\title{
PERIODIC ORBITS AROUND A MASSIVE STRAIGHT SEGMENT
}

\author{
ANDRÉS RIAGUAS* \\ TERMA at European Space Operations Centre. 64293 Darmstadt. Germany \\ ANTONIO ELIPE \\ Grupo de Mecánica Espacial. Universidad de Zaragoza. 50009 Zaragoza. Spain \\ and \\ MARTÍN LARA \\ Real Observatorio de la Armada. 11110 San Fernando. Spain
}

\begin{abstract}
In this paper, we consider the motion of a particle under the gravitational field of a massive straight segment. This model is used as an approximation to the gravitational field of irregular shaped bodies, such as asteroids, comet nuclei and planets's moons. For this potential, we find several families of periodic orbits and bifurcations.
\end{abstract}

\section{Introduction}

The interest for studying orbits around small celestial bodies such as asteroids and nuclei comets has grown considerably in the recent years. The most important space agencies have included missions to such small celestial bodies in their current programs, like the NEAR and ROSETTA missions $(13 ; 17)$, just to mention but a few. These missions consider the flying of a spacecraft around an asteroid and even the landing on its surface (ROSETTA). On the other hand, in binary asteroids (e.g. Ida and Dactyl), the density of one asteroid may be inferred through the dynamics of its satellite (3).

The classical approach for handling the gravitational potential of a celestial body is to expand it in series of spherical harmonics (see e.g. (9), (8)); when the shape of the body is an spheroid, the convergence of the series is fast enough. However, when the shape is irregular, which happens in many of the celestial objects (asteroids, comet nuclei or planets' satellites like Phobos), these series hardly converge in the vicinity of the body. New models that fit better the main shape features of the body must be used instead.

When irregular shaped bodies are considered, such as the asteroids Eros, Ida, Amaltea (J5), etc., we found the body elongation as their main shape feature. This elongated shape makes pseudospherical approach to the gravitational field of this mass distribution far from the true effect. Indeed, the series expansion of the gravitational potential has its convergence guaranteed outside any sphere centered at the center of mass of the body and radius such that it encloses completely the mass of the body; hence, in the cases of elongated bodies there is a gap where the representation of the field force is uncertain.

* On leave from the Dpto. de Matemática Aplicada and Grupo de Mecánica Espacial. Universidad de Zaragoza. 50009 Zaragoza. Spain 
Some alternative models to the expansion in spherical harmonics have already been proposed. For instance, Werner $(18)$ and Scheeres et al. $(15 ; 16 ; 19)$ use the potential and force of an homogeneous polyhedron close in shape to the asteroid. Prieto and Gómez-Tierno (14) model this type of bodies by a massive dipole; they find also that an axial symmetric body can be replaced by a massive wire lying in the axis of symmetry with appropriate mass distribution. Halamek (7) also studies the gravitational field of a massive straight segment.

In this paper, we consider the gravitational field originated by a massive straight segment that is fixed in the space. For this body, we express the potential function in closed form. Once the potential is obtained, we compute families of periodic orbits by means of a generalization of the method of numerically continuation of periodic orbits with respect to a parameter given by Deprit and Henrard (5). As an starter of the method, we compute Poincaré sections. We found several families and their bifurcations.

\section{Potential of a Straight Segment}

Let us consider a straight segment of length $2 \ell$ and mass $M$. The gravitational potential per unit mass created by this one dimensional body at a certain point $P$ in space is given by the line integral

$$
U(P)=-G \int_{L} \frac{\mathrm{d} m}{r} .
$$

where $G$ is the Gaussian constant. Assuming the linear mass density $(\sigma)$ to be constant, this quadrature is not difficult to solve, and appears in textbooks on potential theory (9). Here we will use its expression in terms of intrinsic quantities such as is given in (2).

Let us consider an inertial reference frame $O x y z$, and let $\boldsymbol{x}_{1}$, and $\boldsymbol{x}_{2}$ be the position vectors of the end points of the straight segment. The position vector of a point of the segment is

$$
\boldsymbol{x}=\boldsymbol{x}_{1}+\nu\left(\boldsymbol{x}_{2}-\boldsymbol{x}_{1}\right)=\boldsymbol{x}_{1}+\nu \boldsymbol{x}_{12} \text { with } 0 \leq \nu \leq 1,
$$

hence, $r^{2}=\boldsymbol{x} \cdot \boldsymbol{x}=4 \nu^{2} \ell^{2}+\nu\left(r_{2}^{2}-r_{1}^{2}-4 \ell^{2}\right)+r_{1}^{2}$, and the length element is $\mathrm{d} r=2 \ell \mathrm{d} \nu$, where we denote $r_{1}=\left\|x_{1}\right\|, r_{2}=\left\|x_{2}\right\|$, and $2 \ell=\left\|x_{12}\right\|$.

Thus, the above quadrature is

$$
U(P)=-2 G \sigma \ell \int_{0}^{1} \frac{\mathrm{d} \nu}{r}=-G M \int_{0}^{1}\left(4 \nu^{2} \ell^{2}+\nu\left(r_{2}^{2}-r_{1}^{2}-4 \ell^{2}\right)+r_{1}^{2}\right)^{-1 / 2} \mathrm{~d} \nu,
$$

which results is

$$
U(O)=-\frac{G M}{2 \ell} \log \left(\frac{r_{1}+r_{2}+2 \ell}{r_{1}+r_{2}-2 \ell}\right),
$$

equation that depends only on the distances $\left(2 \ell, r_{1}\right.$ and $\left.r_{2}\right)$. 
As it was expected, equation (1) shows that the potential is symmetric with respect to the line containing the segment. This axial symmetry suggests the convenience of using cylindrical coordinates, for by virtue of Noether's theorem, the projection of the angular momentum on this axis (one of the conjugate moments in cylindrical coordinates) is an integral.

\section{Equations of Motion}

Let us assume now that the straight segment is fixed in the space. In that case, we may choose the origin to be at the center of mass of the segment and take the Cartesian axes in such a way that the segment lies along the $x$-axis.

Under these conditions, and denoting $\mu=G M, s=r_{1}+r_{2}$ and $p=r_{1} r_{2}$, the Lagrangian of the motion of a particle of unit mass in cylindrical coordinates $(\rho, \lambda, x)$ is

$$
\mathcal{L}=\frac{1}{2}\left(\dot{\rho}^{2}+\dot{x}^{2}+\rho^{2} \dot{\lambda}^{2}\right)+\frac{\mu}{2 \ell} \log \left(\frac{s+2 \ell}{s-2 \ell}\right),
$$

where, now, $s$ and $p$ are

$$
s=\sqrt{\rho^{2}+(x-\ell)^{2}}+\sqrt{\rho^{2}+(x+\ell)^{2}}, \quad p=\sqrt{\rho^{2}+(x-\ell)^{2}} \sqrt{\rho^{2}+(x+\ell)^{2}} .
$$

Thus, since the coordinate $\lambda$ is cyclic, the conjugate moment $\Lambda=\rho^{2} \dot{\lambda}$ is an integral, and the problem is reduced to only two degrees of freedom.

The equations of motion in cylindrical coordinates are

$$
\ddot{\rho}=\frac{\Lambda^{2}}{\rho^{3}}-\frac{2 \mu s \rho}{p\left(s^{2}-4 \ell^{2}\right)}, \quad \ddot{x}=\frac{-2 \mu x}{s p}, \quad \frac{d}{d t}\left(\rho^{2} \dot{\lambda}\right)=0 .
$$

These are, precisely, the equations of motion we shall use to find periodic orbits.

Besides, from these equations, we can detect some particular motions. Indeed, when $\dot{\lambda} \equiv 0$, the plane of the motion contains the segment; when $x \equiv 0$, the motion takes place on this plane; and when $\rho \equiv 0$, the motion takes place along the $x$-axis (we do not deal here with collision problems, neither with the passing of the particle through the segment). These two last cases are one degree of freedom system (in $\rho$ and $x$ respectively), hence, integrable.

\section{Periodic Orbits}

To obtain information about the solutions and global behavior of the dynamical system we plot Poincaré sections; they will be of some help in finding initial conditions for periodic solutions.

Although the motion takes place in a 3-D space, the fact that the $\Lambda$ is an integral, allows to study the motion on a plane, namely the plane $O x \rho$, that is rotating about 
the axis $O x$ with angular velocity $\dot{\lambda}$. Besides, the system is autonomous (see Eq. (2)), hence the energy $h$

$$
h=\frac{1}{2}\left(\dot{\rho}^{2}+\dot{x}^{2}+\frac{\Lambda^{2}}{\rho^{2}}\right)-\frac{\mu}{2 \ell} \log \left(\frac{s+2 \ell}{s-2 \ell}\right) .
$$

is an integral.

With this, we define the surface of section as $h=$ constant and $x=0, \dot{x}>0$. On this 2-D surface of section, we plot points $(\rho, \dot{\rho})$.

In the case here considered, we take the units of length, time and mass in such a way that $2 \ell=1, \mu=1$ and the energy $h=-1 / 2$. For each plot, we compute typically about 20 orbits of about 100 revolutions. We made several surfaces sections for different values of the integral $\Lambda$.

From the expression of the energy (4), we find that the closed curve in the plane $(\rho, \dot{\rho})$ that bounds the region where the motion is possible satisfies the equation

$$
\dot{\rho}= \pm \sqrt{2 h+\frac{\mu}{\ell} \log \left(\frac{s+2 \ell}{s-2 \ell}\right)-\frac{\Lambda^{2}}{\rho^{2}}},
$$

and easily is checked that itself is solution of the differential system (3).

In Figure 1 we show a Poincare section for $\Lambda=0.5$. It is seen that the whole manifold of solutions for this case is dominated by a central fixed point, on the $\rho$-axis, of the stable elliptic type. This periodic orbit is surrounded by quasi periodic orbits lying on concentric tori. Besides, we detect several fixed points, some of then inside islands and others corresponding to hyperbolic type equilibria. The initial conditions for some of these orbits are listed on Table I.

For these orbits, we computed an index of stability, namely the trace $(\operatorname{Tr}(T))$ of the matrizant of the associated Hill equation at the end of one period (5). As it is well known, if $|\operatorname{Tr}(T)|>2$, the characteristic exponents of the orbit are of the unstable type; if $|\operatorname{Tr}(T)|<2$, they are of the stable type; and $|\operatorname{Tr}(T)|=2$ represents a case of indifferent stability. This stability index appears on the fourth column of the table.

For these initial conditions, we compute the periodic orbits. Note that these orbits are periodic on the meridian plane $(\rho, x)$, but not necessarily in the Cartesian frame. The Figure 2 shows some of these 2D periodic orbits, both in the $\rho x$-plane and in the Cartesian system.

We compute Poincaré sections for several values of the parameter $\Lambda$. They are presented in Figure 3. For high values of $\Lambda$, the motion is strongly dominated by the central periodic orbit. Nevertheless, as $\Lambda$ decreases, several island and elliptic and hyperbolic points appear, such as the ones described above. For $\Lambda=0.4$, the cross section is different; indeed, the central elliptic points has bifurcated, and now it is converted into hyperbolic, and two new elliptic equilibria appear on the $\rho$-axis. But this situation does not last much, since in the plot for $\Lambda=0.35$, a new pitchfork bifurcation occurs, the unstable point being now stable, and two new symmetric 


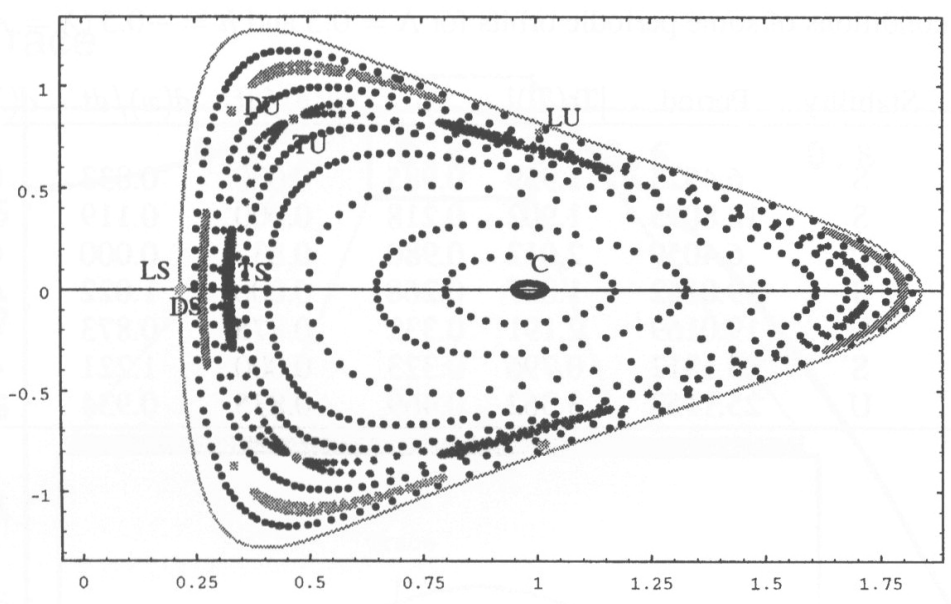

Fig. 1. Poincaré map for $\Lambda=0.5$.
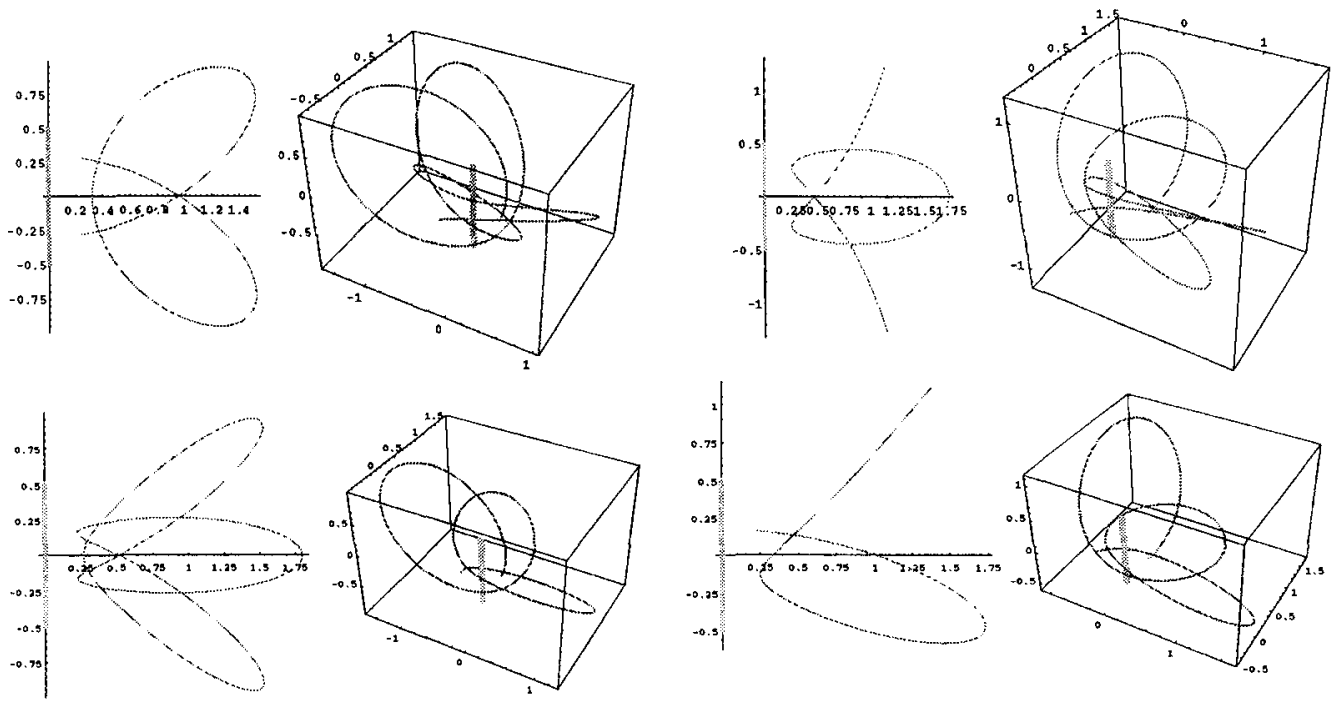

Fig. 2. Periodic orbits in the orbits $\rho x$-plane for $\Lambda=0.5$. Top: TS (left) and TU (right). Bottom: DS (left) and DU (right). The $x y z$ plots are the same orbits in the Cartesian frame. Note that they are not 3D periodic orbits. 
TABLE I

Initial conditions of some periodic orbits for $\Lambda=0.5$ and $h=-0.5(\lambda=x=0)$

\begin{tabular}{lcrrrrrr} 
Orbit & Stability & Period & $|\operatorname{Tr}(T)|$ & $\rho$ & $d(\rho) / d t$ & $d(x) / d t$ & $d(\lambda) / d t$ \\
\hline C & S & 6.4523 & 1.959 & 0.985 & 0.000 & 0.833 & 0.515 \\
LS & S & 12.8095 & 1.902 & 0.218 & 0.000 & 0.119 & 10.51 \\
LU & U & 6.4059 & 2.012 & 0.980 & 0.837 & 0.000 & 0.521 \\
DS & S & 19.0162 & 1.807 & 0.268 & 0.000 & 1.022 & 6.971 \\
DU & U & 19.0169 & 2.191 & 0.332 & 0.875 & 0.873 & 4.515 \\
TS & S & 25.1312 & 0.796 & 0.323 & 0.000 & 1.221 & 4.783 \\
TU & U & 25.1153 & 3.362 & 0.469 & 0.835 & 0.934 & 2.268 \\
\hline
\end{tabular}
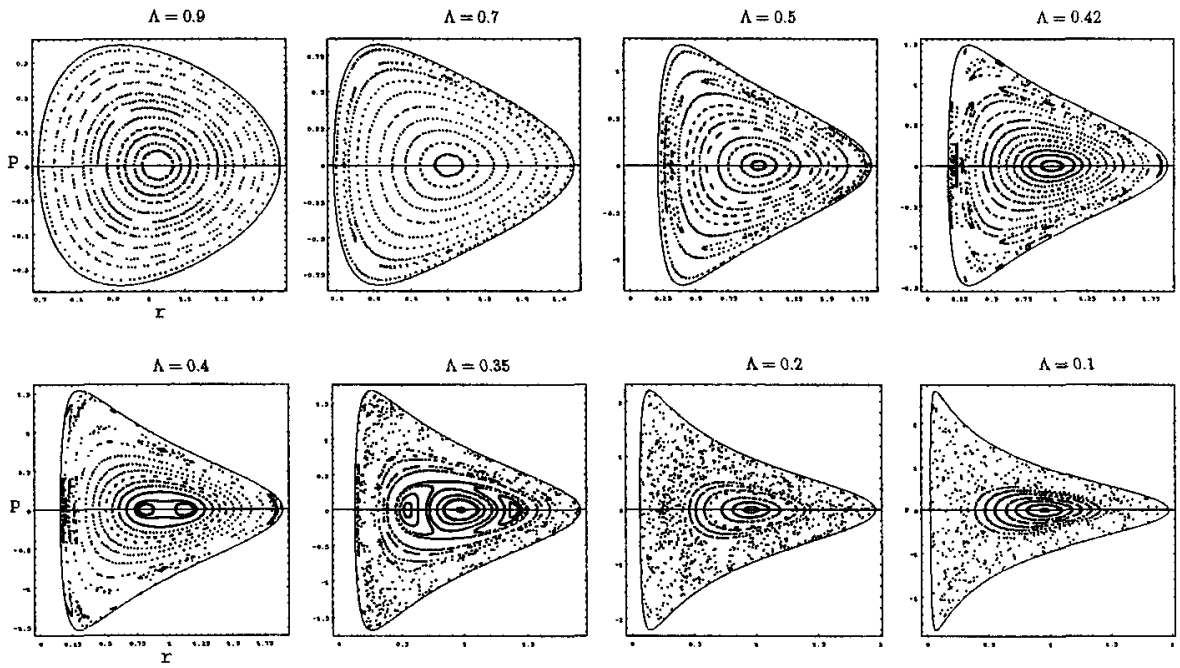

Fig. 3. Poincaré surfaces of section for different decreasing values of the parameter $\Lambda$. The central point corresponding to a periodic orbit can be easily identified.

unstable points appear outside the $\rho$-axis. However, this configuration disappears for $\Lambda=0.2$, where the region of chaotic behavior is wider.

The change of the topology due to the change of stability of the central fixed point and the appearance of a small unstable region along the family of the central fixed points is quite common in other problems, like resonances in asteroids (see e.g. 6) or the critical inclination in artificial satellite theory (see e.g. $4 ; 1$ ). 


\section{Trace}

$\Lambda$

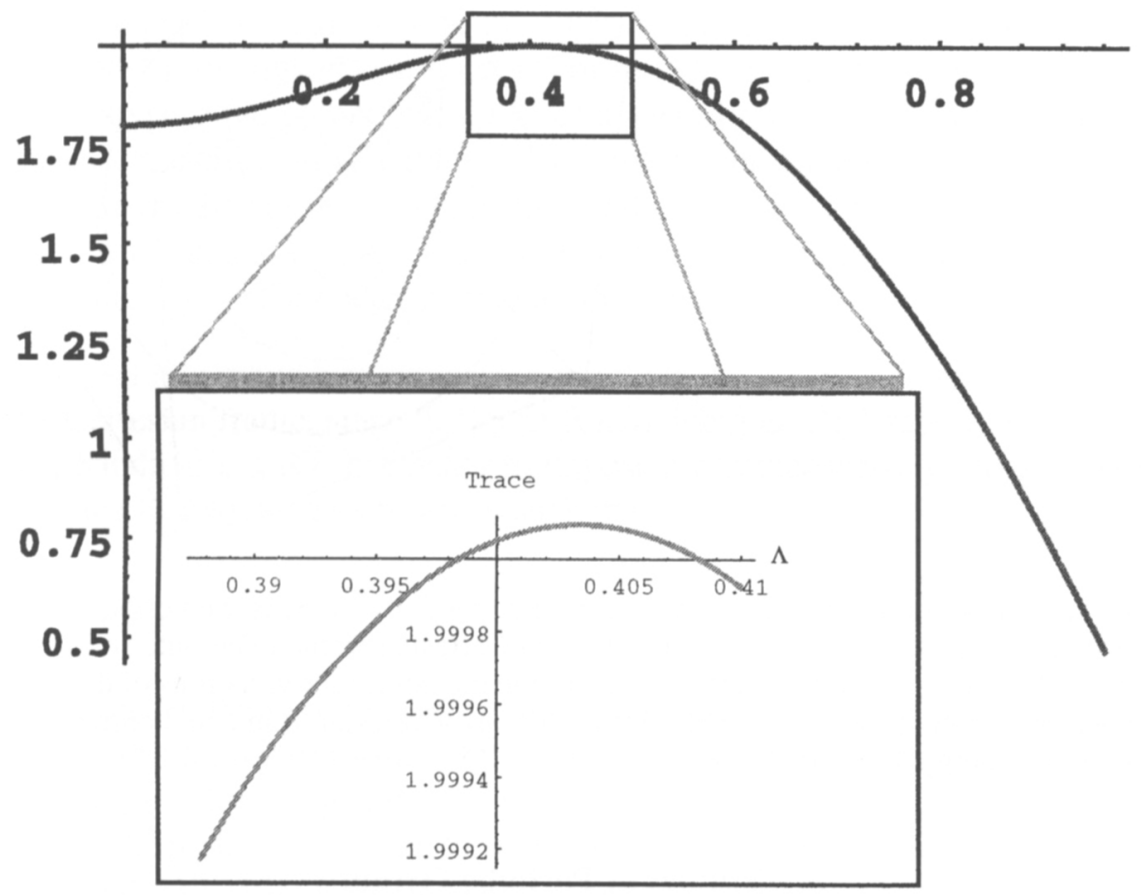

Fig. 4. Evolution of the trace of the matrizant versus $\Lambda$ for the family of circular orbits $(0 \leq \Lambda \leq 1)$. The family is unstable in the interval $0.39844<\Lambda<0.40830$.
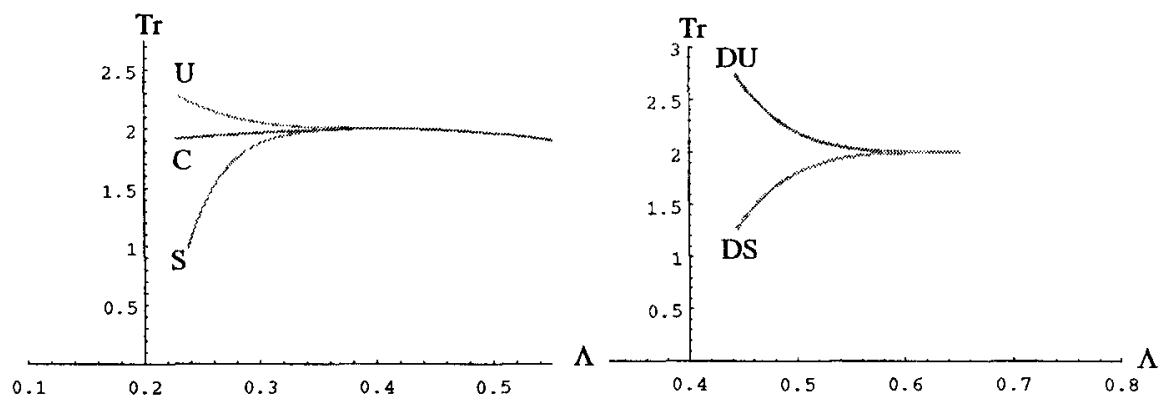

Fig. 5. Trace of the matrizant versus $\Lambda$. Left plot: family of circular orbits and the stable and unstable families that bifurcate from the circular family (the stable family $S$ appears at $\Lambda=0.40830$, and the unstable $U$ at $\Lambda=0.39844$ ). Right plot: families DU (unstable) and DS (stable); these families appear at $\Lambda=0.6492$ and $\Lambda=0.6498$ respectively, and they are bifurcations of another family. 

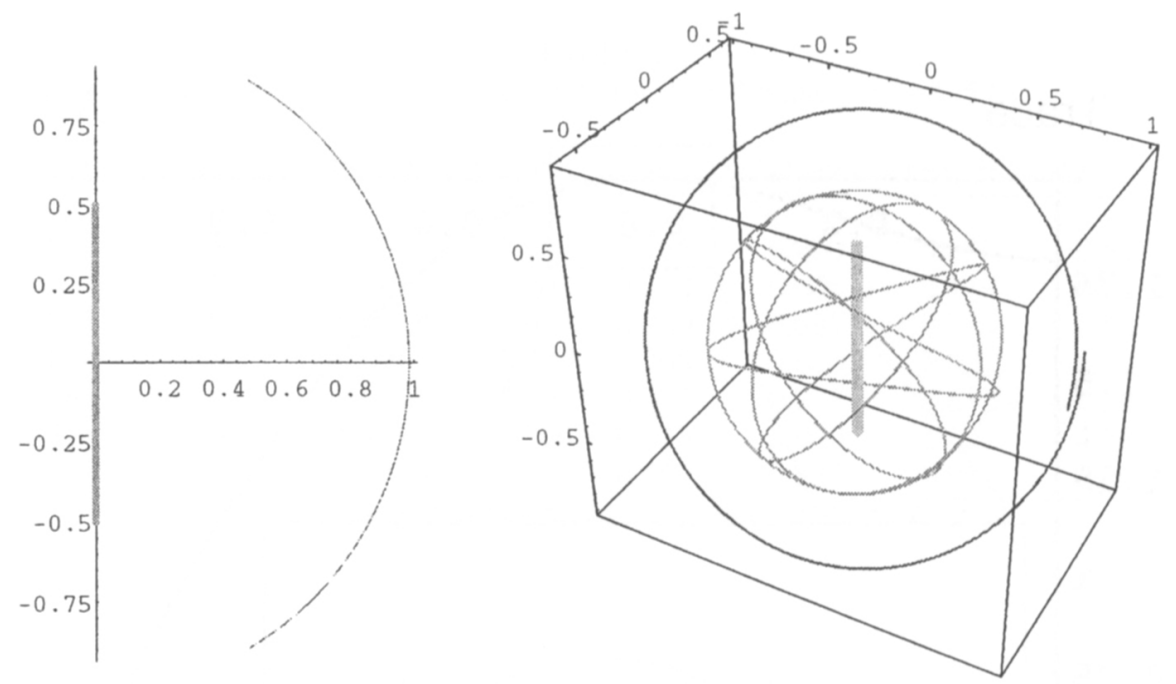

Fig. 6. Circular periodic orbit $\Lambda=0.5$. Left figure (for $h=-0.5$ ) is the periodic orbit in the $\rho x$-plane, that is not a closed orbit in the 3D Cartesian frame (The outer orbit in the right plot). To have a 3D periodic orbit, we continue the family for variations of the energy. The inner orbit (right plot) is a closed orbit in the $x y z$-Cartesian frame and correspond to a value of the energy $h=-0.78728$

\section{Families of Periodic Orbits}

In order to determine the exact values of the parameter $\Lambda$ at which bifurcations occur, we use the method of numerical continuation with respect to a parameter of families of periodic orbits. The method is essentially the one given by Deprit and Henrard 5 with some additions made in Lara et al. 10;11. The process addresses a Boundary Value Problem for the variational equations relative to conservative dynamical systems with two degrees of freedom.

Briefly, it consists in the following: starting with a set of initial conditions close to one periodic solution, we correct this initial set to obtain initial conditions for a true periodic orbit. Then, we vary the value of the parameter ( $\Lambda$ in our case), and by calculating and refining a tangent prediction we obtain new initial conditions corresponding to a periodic orbit for the new value of the chosen parameter. In order to improve the prediction, we must numerically integrate the equations of motion and their tangent and normal variations, the variational equations associated with this solution. The main feature of this method, is that it splits the normal displacements along an orbit from the tangent ones: the latter, indeed, are secular in nature. For details, the reader is addressed to $5 ; 10$.

We computed the family starting with the circular orbit $\mathrm{C}$. For each periodic orbit belonging to this family, after one period $T$, we determine the trace $(|\operatorname{Tr}(T)|)$ of the associated Hill equation. The transition from $|\operatorname{Tr}(T)|<2$ to $|\operatorname{Tr}(T)|>2$ (and 
conversely) will correspond to the bifurcation. In Figure 4 we present the evolution of the trace $|\operatorname{Tr}(T)|$ versus the parameter $\Lambda$. At the value $\Lambda=0.40830$ we have the first pitchfork bifurcation; the elliptic point in the Poincaré section is split into three points, one hyperbolic and two of the elliptic type. The next pitchfork happens for $\Lambda=0.39844$; the hyperbolic point is converted, again, into an elliptic point and two new hyperbolic points (with $\rho \neq 0$ ) appear. From the evolution of the trace, we can see the bifurcation and the stability of the three families (Figure 5, left).

Similar results are obtained for the families DS and DU. These families exist for $\Lambda<0.6498$, and they are bifurcations of another family (Figure 5, right). For small values of the parameter $\Lambda$, the families enter into chaotic regions.

The periodic orbits just found are periodic in the $\rho x$-plane, but not necessarily in the Cartesian frame, since the angle $\lambda$ is moving at the $\lambda$ rate. In order to have a $3 \mathrm{D}$ periodic orbit, it is necessary to impose a commensurately relation, namely, there must be two integers $m$ and $n$ such that

$$
\lambda\left(t_{0}+T\right)-\lambda\left(t_{0}\right)=\frac{m}{n} 2 \pi .
$$

Thus, if we fix the two integers $m$ and $n$, we have to find the new period of the periodic orbit for a given value of the parameter $\Lambda$ in order to fulfill the periodicity condition (5). This is equivalent to find periodic orbits that repeat its ground track 12. Since the period varies with the altitude - that depends on the energy, and since our method for continuation of natural families is valid for whatever parameter, we compute the natural families of circular periodic orbits for variation of the energy $h$, that now is taken as a parameter. As an illustration, we compute the 3D circular orbit for $n=5, m=1$ and $\Lambda=0.5$. As starter, we use the circular orbit found for $\Lambda=0.5$ and $h=-0.5$ (see Table $\mathrm{I}$ ). By varying the energy, we found that the repetition condition (5) is fulfilled for $h=-0.78728$, to which corresponds a circular orbit (see Figure 6) with initial conditions $\rho=0.648485, x=0.818833$, $\rho=x=0$ and the period is $T=3.885305$.

\section{Conclusions}

The gravitational field of a very elongated celestial body is modeled by a massive straight segment fixed in the space. For this logarithmic potential we found families of periodic orbits and their bifurcations. Work for a rotating segment is in progress.

\section{Acknowledgements}

Thanks are due to Professor Broucke for suggesting us this problem. Comments from an anonymous referee improved the final version of the manuscript. Partial support comes from the Spanish Ministry of Education (CICYT \# PB95-0807). 


\section{References}

Broucke, R.: 1994, "Numerical Integration of Periodic Orbits in the Main Problem of Artificial Satellite Theory," Celest. Mech. \& Dynam. Astr., 58, 99-123.

Broucke, R.: 1995, "Closed form expressions for some gravitational potentials: triangle, rectangle, pyramid and polyhedron." AAS/AIAA Spaceflight Mechanics Meeting, AAS 95-190, Albuquerque.

Byrnes, D.V., D'Amario, L.A.: 1995, "Dactyl orbit determination analysis," AAS/ALAA Astrodynamics Specialist Conference, AAS 95-315, Halifax.

Coffey, S. L., Deprit, A., Miller, B. R.: 1986, "The Critical Inclination in Artificial Satellite Theory," Celest. Mech. \& Dynam. Astr., 39, 365-406.

Deprit, A. and Henrard, J.: 1967, "Natural families of periodic orbits," Astronomical Joumal 72, $158-172$.

Hadjidemetriou, J.D.: 1993, “Asteroid Motion near the 3:1 Resonance," Celest. Mech. \& Dynam. Astr., 56, 563-599.

Halamek, P.: 1988, Motion in the potential of a thin bar. $\mathrm{Ph}$. D. dissertation. Univ. of Texas. Austin.

Heiskanen, W. A., Moritz, H.: 1967, Physical Geodesy, W. H. Freeman and Co., San Francisco.

Kellogg, O. D.: 1954, Foundations of Potential Theory. Dover Publications, Inc. New York.

Lara, M., Deprit, A., Elipe, A.: 1995, "Numerical continuation of frozen orbits for the zonal problem." Celest. Mech. \& Dynam. Astr., 62, 167-181.

Lara, M.: 1996, "On numerical continuation of families of periodic orbits in a parametric potential." Mechanics Research Communications, 23, 291-298.

Lara, M.: 1997, "On periodic polar orbits on the Artificial Satellite Problem." Journal of the Astronautical Sciences, 45, 321-328.

"NEAR.- Special Issue on the NEAR Mission to 433 Eros": 1995, Journal Astronautical Sciences 43.

Prieto-Llanos, T., Gómez-Tierno, M.A.: 1994, "Station keeping at libration points of natural elongated bodies," Journal of Guidance Control and Dynamics, 14, 787-794.

Scheeres, D.J.: 1995, "Satellite dynamics about Eros," AAS/AIAA Spaceflight Mechanics Meeting, AAS 95-110, Albuquerque.

Scheeres, D.J., Ostro, S.J., Hudson, R. S., Werner, R. A.: 1996, “Orbits close to Asteroid 4769 Castalia," Icarus, 121, 67-87.

Schwehm, G., Hechler, M.: 1994, "Rosetta - ESA's Planetary cornerstone mission," ESA Bulletin, 77, 7-18.

Werner, R. A.: 1994, "The gravitational potential of a homogeneous polyhedron or don't cut corners," Celest. Mech. \& Dynam. Astr., 59, 253-278.

Werner R.A., Scheeres D.J.: 1996 , "Exterior gravitation of a polyhedron derived and compared with harmonic and mascon gravitation representation of asteroid 4769 Castalia," Celest. Mech. \& Dynam. Astr., 65, 313-344. 